\title{
Direct Flow valve-in-valve implantation in a degenerated mitral bioprosthesis
}

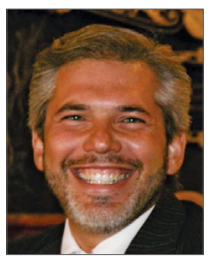

Giuseppe Bruschi ${ }^{1 *}$, MD, FESC; Aldo Cannata ${ }^{1}, \mathrm{MD}$; Alberto Barosi ${ }^{1}, \mathrm{MD}$;

Paola Colombo ${ }^{1}, \mathrm{MD}$, PhD; Francesco Soriano ${ }^{1}, \mathrm{MD}$; Stefano Nava ${ }^{1}, \mathrm{MD}$; Elisa Montrasio², MD; Luca Botta ${ }^{1}, \mathrm{MD}, \mathrm{PhD}$; Maria Pia Gagliardone², MD; Silvio Klugmann ${ }^{1}$, MD; Federico De $\mathrm{Marco}^{1}, \mathrm{MD}, \mathrm{PhD}$

1. Cardiology \& Cardiac Surgery Department “A. De Gasperis”, Niguarda Ca' Granda Hospital, Milan, Italy; 2. Cardiothoracic Anesthesia and Intensive Care, Niguarda Ca' Granda Hospital, Milan, Italy

This paper also includes supplementary data published online at: http://www.pcronline.com/eurointervention/97th_issue/298

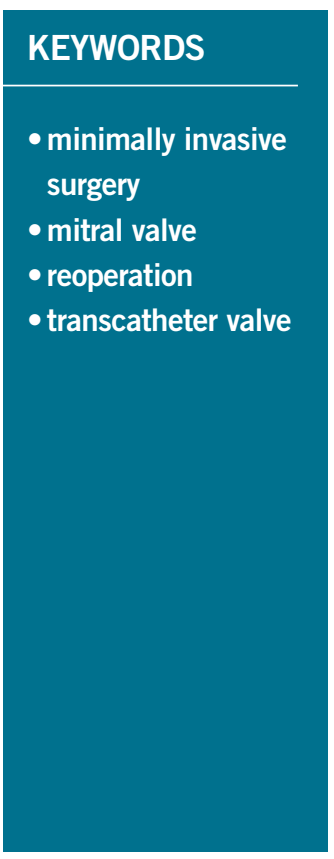

\section{Abstract}

Aims: Mitral valve reoperations due to failing bioprostheses, in patients affected by multiple comorbidities, are associated with high morbidity and mortality. Transcatheter techniques may evolve as complementary approaches to surgery in these patients at high risk for surgery.

Methods and results: We describe a case of Direct Flow $25 \mathrm{~mm}$ transcatheter valve implantation as valvein-valve in a degenerated mitral bioprosthesis through a transapical approach in a 63-year-old man affected by dilated cardiomyopathy. The patient was affected by Carpentier-Edwards $29 \mathrm{~mm}$ severe regurgitation. The $25 \mathrm{~mm}$ Direct Flow bioprosthesis was advanced through the mitral bioprosthesis into the left atrium and then positioned using the three independent positioning wires. Transoesophageal echocardiography evidenced normal Direct Flow function with no paravalvular regurgitation and a low transmitral gradient of $4 \mathrm{mmHg}$.

Conclusions: Our successful experience, characterised by a Heart Team approach and multidisciplinary patient care, demonstrated the technical feasibility and procedural safety of Direct Flow valve-in-valve mitral implantation.

\footnotetext{
*Corresponding author: Cardiology \& Cardiac Surgery Department “A. De Gasperis”, Niguarda Ca' Granda Hospital, Piazza dell'Ospedale Maggiore 3, 20162 Milan, Italy.E-mail: giuseppe.bruschi@fastwebnet.it
} 
Transcatheter mitral valve-in-valve implantation into failing surgical bioprosthetic valves has been reported previously. It has evolved in recent years as a promising complementary therapy to avoid repeat cardiac surgery in patients affected by multiple comorbidities and who are considered at high risk for surgical reoperation $^{1,2}$. Different approaches to access the mitral bioprosthesis or ring have been described, including the transseptal and transatrial techniques. Nevertheless, most experience has been gained using the transapical access ${ }^{3-5}$.

The current standard when treating degenerated mitral bioprostheses with transcatheter valves is the use of Edwards balloonexpandable devices (Edwards Lifesciences, Irvine, CA, USA). This technique gives good overall results but it is limited by lack of repositionability and retrievability and the issues associated with residual regurgitation and malpositioning. The Direct Flow Medical ${ }^{\circledR}$ Transcatheter Aortic Valve System (Direct Flow Medical Inc., Santa Rosa, CA, USA) has the potential to overcome these limitations.

We describe a case of Direct Flow Medical transcatheter valve implantation as valve-in-valve in a degenerated mitral bioprosthesis through the transapical approach.

The Direct Flow Medical device is the first transcatheter valve device which is not based on a metallic frame technology. The bovine pericardial prosthesis has an inflatable and deflatable support structure that allows precise positioning, repositioning, and retrieval if needed.
Full assessment of the valve performance in its intended final configuration can be carried out with multiple modalities, including complete echographic assessment, before permanent fixation with a durable polymer ${ }^{6}$.

\section{Case report}

A 63-year-old man (height: $185 \mathrm{~cm}$, weight: $135 \mathrm{~kg}$, body mass index: $39.4 \mathrm{~kg} / \mathrm{m}^{2}$ ) affected by dilated cardiomyopathy was admitted to our department for worsening dyspnoea at rest. His symptoms were consistent with New York Heart Association Class IV. In 2008, the patient had undergone coronary artery revascularisation and concomitant mitral valve replacement with a CarpentierEdwards $29 \mathrm{~mm}$ bioprosthesis (Edwards Lifesciences). In 2012, he experienced a non-ST-elevation myocardial infarction treated by percutaneous coronary intervention. His comorbid conditions included obstructive sleep apnoea syndrome treated with nocturnal continuous positive airway pressure therapy, chronic atrial fibrillation, pulmonary hypertension and moderate chronic kidney disease. In recent years, the patient had had several hospital admissions for heart failure with evidence of mitral bioprosthetic dysfunction.

Echocardiography on admission (Figure 1A) confirmed left ventricular (LV) dysfunction with an LV ejection fraction of $35 \%$ and severe bioprosthetic mitral stenosis (valve area, $0.7 \mathrm{~cm}^{2}$; mean gradient, $10 \mathrm{mmHg}$ ). This was associated with severe regurgitation and an elevated pulmonary artery systolic pressure of $60 \mathrm{mmHg}$.

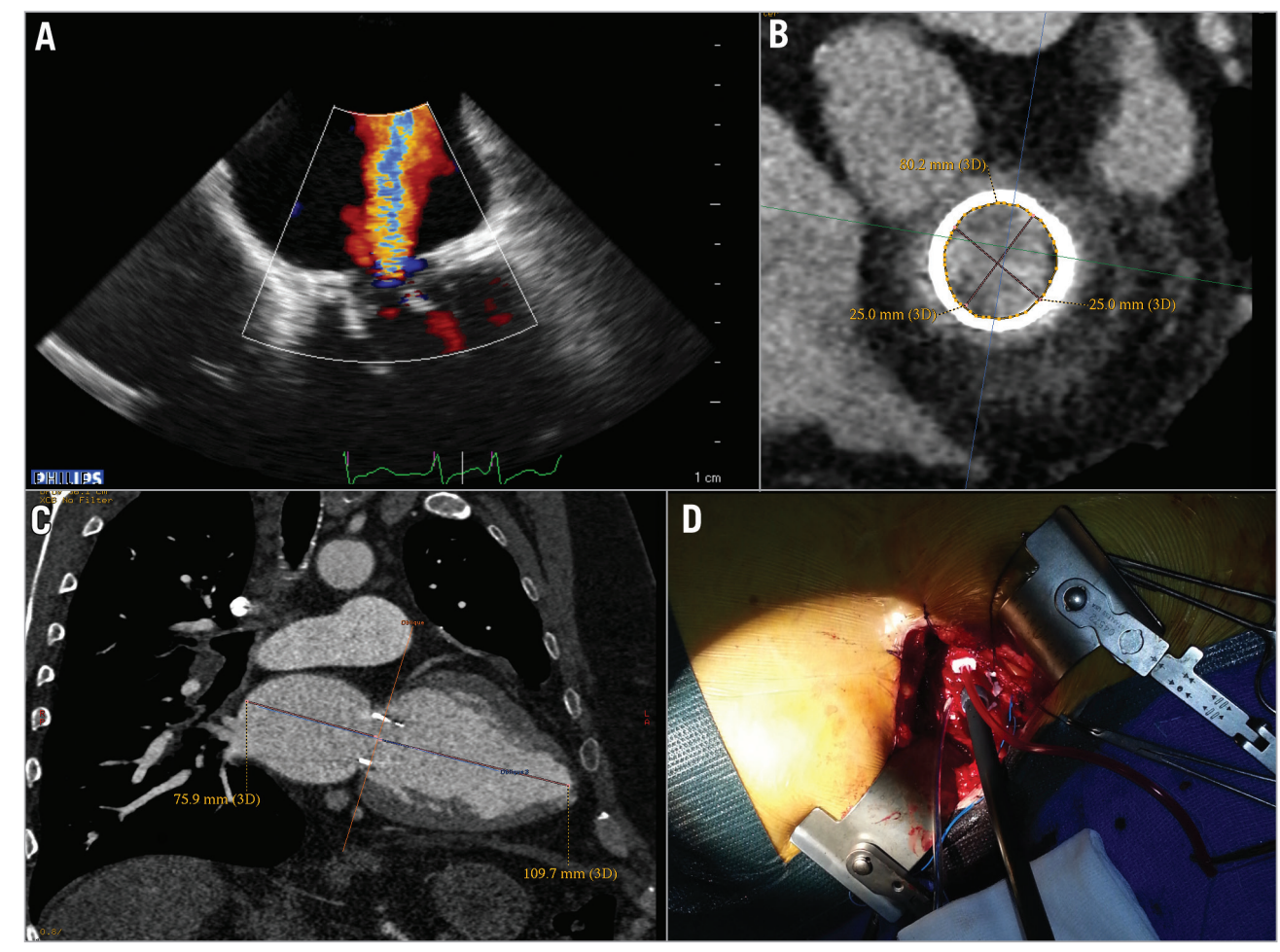

Figure 1. Echocardiographic and CT scan mitral bioprosthesis evaluation. A) Mitral bioprosthesis severe regurgitation. B) CT scan mitral bioprosthesis evaluation at the level of the sewing ring, diameter $25 \mathrm{~mm}$ and perimeter of $80.2 \mathrm{~mm}$. C) CT scan evaluation of the space in the left atrium to unsheathe the Direct Flow bioprosthesis. D) Transapical insertion of a Check-Flo Cook sheath for Direct Flow valve deployment. 
According to our institutional policy the patient was jointly evaluated by our Heart Team, adopting the same philosophy we use for transcatheter aortic valve implantation. A transcatheter mitral valvein-valve procedure was considered since the patient was a high-risk surgical candidate due to his comorbidities and previous cardiac surgery (EuroSCORE II: 24.59\%, logistic 47\%; and Society of Thoracic Surgeons risk of mortality $27.1 \%$, and morbidity and mortality $62 \%$ ).

After careful patient and procedural evaluation, and on the basis of our own and other authors' experience of Edwards SAPIEN valve migration or severe paravalvular regurgitation after mitral valve-in-valve procedures ${ }^{1,7,8}$, we decided to perform a Direct Flow Medical device implantation through the transapical approach. Detailed written informed consent was obtained from our patient.

The procedure was performed in our hybrid suite by our Heart Team composed of cardiac surgeons with expertise in hybrid procedures, interventional cardiologists, cardiac anaesthesiologists and echocardiographists.

The procedure was performed under general anaesthesia and double lumen intubation to allow single lung ventilation. Defibrillator pads were properly placed across the chest wall. During the procedure, unfractionated heparin $100 \mathrm{IU} / \mathrm{kg}$ was administered to achieve an activated clotting time of 200 to 250 seconds for the duration of the procedure. This was then reversed with protamine at the end of the operation. A 6 Fr sheath was inserted in the left femoral artery for haemodynamic monitoring and one in the right femoral vein to have the possibility to establish a femoro-femoral emergency cardiopulmonary bypass support in case of haemodynamic collapse due to procedural issues. A standard transapical approach was performed by left anterolateral mini-thoracotomy at the fifth intercostal space; the pericardium was dissected from the epicardium to expose the LV apex. After coaxial trajectory evaluation, between entry site and mitral bioprosthesis (Figure 1B, Figure 1C), two paired orthogonal U-shaped sutures with pledgets were placed to secure the apex. Two temporary epicardial pacing leads were placed on the LV. The left ventricle was accessed using the Seldinger technique through the double sutures, a 12 Fr sheath was inserted and mitral bioprosthesis crossed with a straight wire. After replacement of the soft guidewire by an Amplatz Super-Stiff ${ }^{\text {TM }}$ (Cook Medical, Bloomington, IN, USA), positioned across the mitral bioprosthesis into the right lower pulmonary vein, a $24 \mathrm{Fr}$ sheath was advanced to deploy the Direct Flow device (Figure 1D). Mitral prosthesis balloon valvuloplasty was performed under rapid pacing using a Loma Vista Medical TRUE Dilatation ${ }^{\mathrm{TM}}$ Balloon Valvuloplasty Catheter, $24 \mathrm{~mm}$ (Bard Medical, Covington, GA, USA). A $25 \mathrm{~mm}$ Direct Flow bioprosthesis was advanced through the mitral bioprosthesis into the left atrium (Figure 2A). After unsheathing, the valve rings were inflated at 12 atmospheres in the left atrium with a contrast-saline mixture through the positioning wires of the delivery system to allow for valve unfolding. The bioprosthesis was then positioned using the three independent positioning wires with a modified inner curve technique in a similar way to that

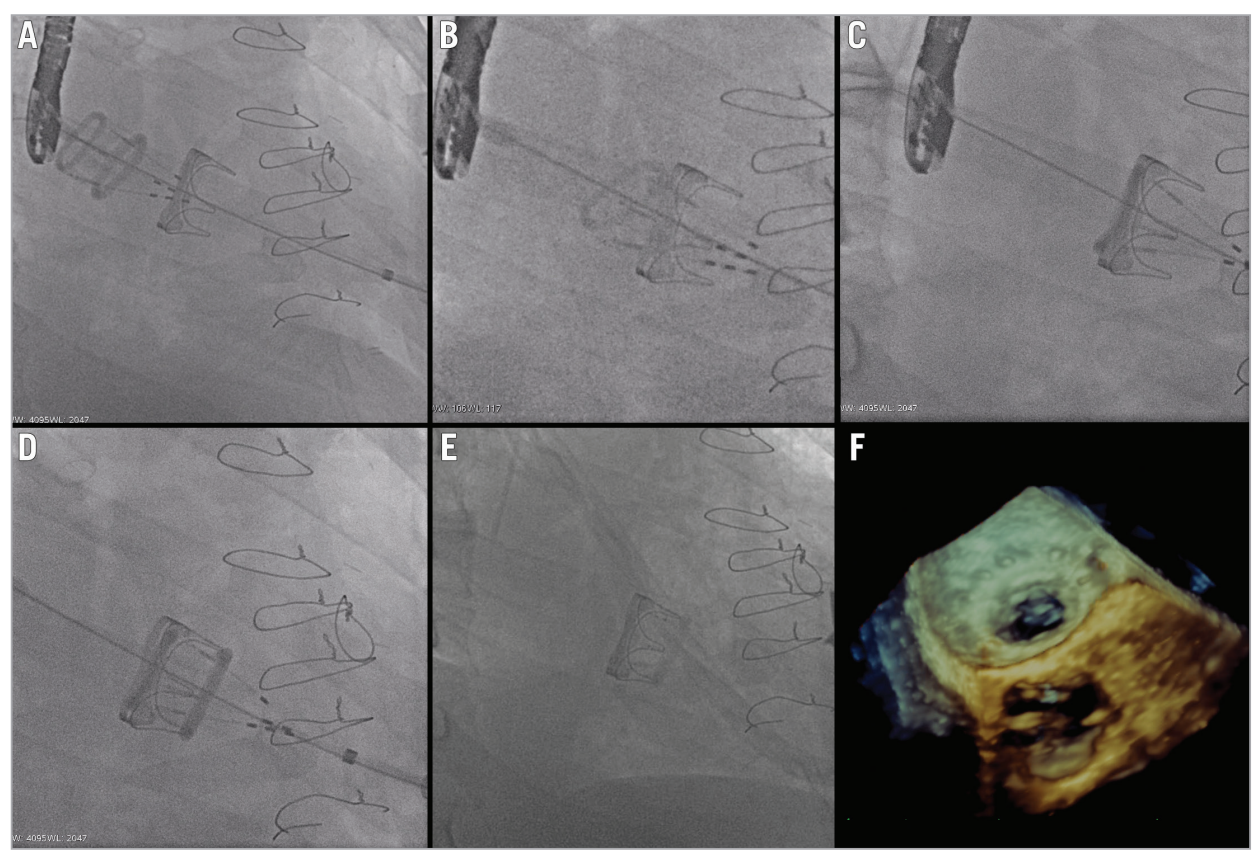

Figure 2. Direct Flow valve-in-valve mitral implantation. A) Direct Flow was unsheathed in the left atrium and valve rings were inflated at 12 atmospheres to unfold the valve. B) The bioprosthesis was then positioned using the three independent positioning wires with a modified inner curve technique. C) Atrial ring was positioned inside the bioprosthetic stent at the level of the sewing ring. D) Direct Flow ventricular ring was positioned on the top of the tip of stent posts of the Carpentier-Edwards bioprosthesis. E) Final Direct Flow position after positioning wires have been detached. F) 3D echocardiographic image of the atrial Direct Flow ring positioned inside the bioprosthetic stent at the level of the sewing ring. 
which is routinely carried out during TAVI with the Direct Flow device $^{9}$ (Figure 2B-D). Some minimal repositioning performed by deflating only the larger (ventricular) ring and pulling on the positioning wires was carried out in order to position the ventricular ring on the top of the tip of stent posts. Fluoroscopic optimal expansion of the Direct Flow valve inside the Carpentier-Edwards bioprosthesis was confirmed by the symmetric and circular inflation of the atrial ring inside the bioprosthetic stent at the level of the sewing ring (Figure 2E). Transoesophageal echocardiography evidenced normal Direct Flow function (Figure 2F) with no paravalvular regurgitation and a low transmitral gradient of $4 \mathrm{mmHg}$. The contrast-saline mixture was then exchanged for the polymer to allow permanent valve fixation. Positioning wires were then detached and the delivery system removed. LV sutures were knotted regularly, thoracotomy was closed in the standard fashion, and the patient was transferred to the intensive care unit for recovery. He was extubated 48 hours after the procedure. The patient was discharged home on the ninth postoperative day, thoracotomy wound heal by primary intention.

Transcatheter mitral valve-in-valve implantation with the Edwards SAPIEN balloon-expandable device has proved to be an attractive alternative to conventional reoperation for elderly high-risk surgical patients with bioprosthetic degeneration. Nevertheless, in the literature, cases of delayed valve migration have been reported together with cases of significant residual regurgitation, mainly related to the choice of size of the TAVI device or its suboptimal placement unable to prevent embolisation $^{1,7,8}$. In our case we implanted a Direct Flow $25 \mathrm{~mm}$ device in a Carpentier-Edwards Perimount $29 \mathrm{~mm}$ bioprosthesis. Valve size was chosen mainly relying on computed tomography sizing (Figure 1B) and not on the internal diameter reported by the manufacturer. The internal diameter of the Perimount at the level of the sewing ring on CT scan measured $25 \mathrm{~mm}$ (derived from a perimeter of $80.2 \mathrm{~mm}$ ). Since the Direct Flow device in the aortic location does not require significant oversizing, we opted for a 1:1 ratio between the inner diameter of the Perimount and that of the atrial ring of the Direct Flow device. Our assumption was that the shape of the Direct Flow device with the unique double ring design of the valve could create a double seal - both around the atrial portion of the bioprosthesis and also at the level of the tip of stent posts of the mitral bioprosthesis. Moreover, we speculated that the larger ventricular ring expanded beyond the tip of the stent posts could prevent delayed atrial embolisation of the TAVI device. This is purely a hypothesis. Obviously no definite conclusions about a rare complication such as delayed migration can be drawn from a single case.

Size choice might appear conservative as we selected a $25 \mathrm{~mm}$ device for a device having an inner diameter of $27 \mathrm{~mm}$. Being a retrievable device, we chose to start with a relatively small size. In case of a suboptimal result, the planned strategy would have been that of retrieving it and upsizing to a $27 \mathrm{~mm}$ Direct Flow Medical (DFM) device. This did not happen due to the acceptable haemodynamics obtained with the first device. If retrieval had been carried out, it would have been done according to a modified technique to retrieve the device in very limited spaces which has been tested effectively on the bench. Basically, the two main differences from the standard technique are: using a larger sheath so that less force is needed when extracting the device, and opening the retrieve basket inside the sheath and carefully advancing it from its tip to "encage" the valve instead of opening the basket inside the body and pulling the valve inside the basket.

Our successful experience, characterised by a Heart Team approach and multidisciplinary patient care, demonstrated the technical feasibility and procedural safety of Direct Flow valve-invalve mitral implantation.

\section{Impact on daily practice}

A less invasive approach for mitral re-replacement is desirable not only for a high-risk elderly population. Transcatheter mitral valve-in-valve implantation for a dysfunctional biological mitral prosthesis can be performed with low operative mortality. Even if further improvements are needed before widespread clinical application, significant clinical impact can be anticipated, not only because the absolute number of failing mitral biological prostheses will continue to grow, but also because a change in the surgical community should be anticipated, favouring mitral valve replacement with a bioprosthesis to treat functional mitral regurgitation instead of repairing the valve.

\section{Conflict of interest statement}

G. Bruschi is a consultant for Medtronic and Direct Flow. F. De Marco is a consultant for Direct Flow. The other authors have no conflicts of interest to declare.

\section{References}

1. Cheung A, Webb JG, Barbanti M, Freeman M, Binder RK, Thompson C, Wood DA, Ye J. 5-year experience with transcatheter transapical mitral valve-in-valve implantation for bioprosthetic valve dysfunction. J Am Coll Cardiol. 2013;61:1759-66.

2. Seiffert M, Conradi L, Baldus S, Schirmer J, Knap M, Blankenberg S, Reichenspurner H, Treede H. Transcatheter mitral valve-in-valve implantation in patients with degenerated bioprostheses. JACC Cardiovasc Interv. 2012;5:341-9.

3. Cheung A, Webb JG, Wong DR, Ye J, Masson JB, Carere RG, Lichtenstein SV. Transapical Transcathter Mitral Valve-in-Valve Implantation in a Human. Ann Thorac Surg. 2009;87:e18-20.

4. Schaefer U, Frerker C, Schewel D, Thielsen T, Meincke F, Kreidel F, Kuck KH. Transfemoral and transseptal valve-in-valve implantation into a failing mitral xenograft with a balloonexpandable biological valve. Ann Thorac Surg. 2012;94:2115-8.

5. Bruschi G, Barosi A, Colombo P, Botta L, Oreglia J, De Marco F, Paino R, Klugmann S, Martinelli L. Direct transatrial transcatheter SAPIEN valve implantation through right minithoracotomy in a degenerated mitral bioprosthetic valve. Ann Thorac Surg. 2012;93:1708-10. 
6. Schofer J, Colombo A, Klugmann S, Fajadet J, DeMarco F, Tchétché D, Maisano F, Bruschi G, Latib A, Bijuklic K, Weissman N, Low R, Thomas M, Young C, Redwood S, Mullen M, Yap J, Grube E, Nickenig G, Sinning JM, Hauptmann KE, Friedrich I, Lauterbach M, Schmoeckel M, Davidson C, Lefevre T. Prospective multicenter evaluation of the direct flow medical transcatheter aortic valve. J Am Coll Cardiol. 2014;63:763-8.

7. Bruschi G, Botta L, Fratto P, Martinelli L. Failed valve-invalve transcatheter mitral valve implantation. Eur J Cardiothorac Surg. 2014;45:e127.

8. Bapat VN, Khaliel F, Ihleberg L. Delayed migration of Sapien valve following a transcatheter mitral valve-in-valve implantation. Catheter Cardiovasc Interv. 2014;83:E150-4.

9. De Marco F, Latib A. Tools and Techniques - Clinical: the inner curve technique for implantation of the Direct Flow Medical ${ }^{\circledR}$ transcatheter aortic valve. EuroIntervention. 2014;10:400-2.

\section{Supplementary data}

Moving image 1. Mitral bioprosthesis echocardiographic evaluation.

Moving image 2. The Direct Flow valve is advanced through the mitral bioprosthesis in the left atrium and unsheathed.

Moving image 3. The Direct Flow valve was positioned inside mitral bioprosthesis using the three independent positioning wires. Moving image 4. Final adjustment of the Direct Flow position and valve inflation.

Moving image 5. Final Direct Flow valve position. After polymer fixation, the positioning wires were detached.

The supplementary data are published online at:

http://www.pcronline.com/

eurointervention/97th_issue/298 